\title{
Addressing Confusion in Double Star Nomenclature: The Washington Multiplicity Catalog
}

\author{
William I. Hartkopf \& Brian D. Mason \\ U.S. Naval Observatory, Washington, DC
}

(Note: This talk was adapted from one given in February 2003 at IAU Colloquium 191, The Environment and Evolution of Binary and Multiple Stars, in Mérida, México.)

\begin{abstract}
The Washington Multiplicity Catalog (WMC) project is an effort to address the often conflicting nomenclature schemes used by astronomers in different fields of double and multiple star research, by compiling a catalog of all known binary and multiple systems, then assigning a consistent (insofar as possible) component designation to each component. Components are treated in the same manner whether stellar or sub-stellar (e.g., brown dwarf, exosolar planet) in nature. This effort was begun shortly before the 2000 IAU General Assembly in Manchester, UK. A sample version of the planned catalog was presented at IAU Colloquium 191, held in Mérida, México in February 2003, and a modified version of this sample will be presented at the July $2003 \mathrm{GA}$ in Sydney, Australia. Finally, if the nomenclature and catalog format are agreed upon, a full-sky version of the catalog will be created in time for the $2006 \mathrm{GA}$ in Prague.
\end{abstract}

\section{Roots of the WMC}

The root of the Washington Multiplicity Catalog (WMC) is the Washington Double Star Catalog (WDS). The WDS, maintained at the United States Naval Observatory (USNO), is the principal database of astrometric double and multiple star data for the astronomical community. It contains (as of July 2003) 603,651 mean positions for 99,403 pairs, and is updated nightly. The USNO double-star program also maintains catalogs of differential magnitudes, visual orbital elements, and interferometric and other high-resolution observations.

While the WDS is a complete listing of all resolved systems (i.e., visual and interferometric doubles), many components are detected but not resolved. These include:

- spectroscopic doubles (single- or double-lined),

- photometric or eclipsing binaries,

- astrometric doubles,

- lunar occultation doubles,

- other doubles, and

- planets and other sub-stellar objects.

However, the WDS nomenclature rules (with slight modification) can accommodate all types of double stars. 


\section{The WDS and Hierarchy}

The WDS is system-based rather than object-based, as it contains relative measures between components of a given system. In an object-based scheme, a group of $\mathrm{N}$ objects yields $\mathrm{N}$ entries, but in a system-based scheme, $\mathrm{N}$ objects can yield up to $\frac{N(N-1)}{2}$ entries. The WDS may also contain measures between photocenters, and multiple systems may become quite complex as $\mathrm{N}$ increases. However, the WDS lists only pairings actually measured, and the observer's common sense usually implies the system hierarchy.

Generally, orbital period and/or separation are used to assign the hierarchical structure. The 3:1 ratio of semi-major axes determined by R.S. Harrington in his work on hierarchical multiples is generally followed, although separations $>1^{\prime \prime}$ are usually given upper-case letters. This 3:1 ratio assumes physicality. However, most visual doubles do not have enough measures to determine whether motion is Keplerian or rectilinear. In general, then, all hierarchies in the WMC are apparent rather than absolute. It is assumed that all double stars within some small separation are of interest (if only as a warning of possible image blending), so are retained in the WDS and WMC even if shown to be optical.

\section{Rules of Component Designation}

The WDS at present extends nomenclature to second level hierarchies. The WMC will extend this nomenclature to cover more complex systems, however, as follows:

Level 1: capital letters (e.g., STF1523 AB)

Level 2: lower case letters (e.g., FIN $347 \mathrm{Aa}, \mathrm{Ab}$ )

Level 3: numbers (e.g., BNK 1 Ab1,Ab2)

Higher levels will alternate lower case letters and numbers (no examples of higher levels have yet been found, however).

A comma will be used as the delimiter between components in a system, with the full component identifier before and after the comma (e.g., Aa,Ab). The only exception: if only two characters are provided the delimiter is assumed (e.g., WAK 8CD = WAK 8C,D).

While the WMC will strive to maintain hierarchies in the assignment of letter and/or number, this is not always possible, given our often very limited knowledge. Also, if a subsystem is found that cannot be assigned unequivocally to a higher order component, a tentative best guess assignment will be given and a note added to the catalog.

Figure 1 illustrates a (fictitious) system, growing increasingly complex as new components are discovered.

\section{The Sample WMC}

The $11^{h}-11^{h} 30^{m}$ band of RA was selected for the sample WMC. As well as being historically compelling (containing $\xi \mathrm{UMa}$ ), it includes a variety of component types: 


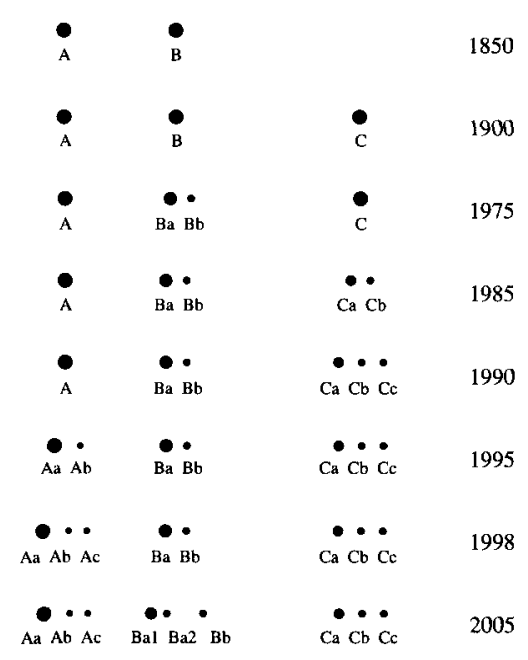

Figure 1. Illustration of nomenclature assignment as a (fictitious) system system grows more complex:

1850: visual pair is discovered

1900: wide common proper motion companion is found

1975: B component is found to be spectroscopic binary

1985: C component is split by speckle interferometry

1990: additional speckle $\mathrm{C}$ component is resolved at a similar separation

1995: planet is found orbiting the A component

1998: second planet is found

2005: primary of B is resolved by long-baseline interferometry

- astrometric binaries,

- X-ray binaries, cataclysmic variables, and related objects,

- eclipsing binaries,

- occultation binaries,

- spectroscopic binaries (SB1 + SB2),

- spectrum binaries,

- interferometric binaries,

- visual binaries, and

- planetary companions (by extending to $10^{h} 59^{m} .5$ ).

In addition to the WDS and other USNO double star catalogs mentioned earlier, sources of multiplicity tapped thus far for the sample WMC include:

- Downes et al., 2001, A Catalog and Atlas of Cataclysmic Variables: On-line Version, PASP 113, 764 (http://icarus.stsci.edu/〜downes/cvcat/),

- Ritter \& Kolb 1998, Catalogue of cataclysmic binaries, low-mass X-ray binaries, and related objects (Sixth edition), A\&AS 129, 83, 
- Batten et al., 1989, Eighth Catalog of the Orbital Elements of Spectroscopic Binary Systems, Pub. DAO, 17,

- Pourbaix et al., 2003, Ninth Catalogue of Spectroscopic Binary Orbits (http://sb9.astro.ulb.ac.be/),

- Svechnikov \& Bessonova 1984, Catalog of Orbital elements, Masses and Luminosities of close double stars, Bull. Inf. CDS 26, 99,

- van Paradijs 1995, A Catalogue of X-Ray Binaries, in X-ray Binaries, Lewin et al, eds., Cambridge Univ. Press, ch. 14, pp 536-577,

- California \& Carnegie Planet Search web site (nttp://exoplanets.org/) and links therein.

Information on other prospective sources is needed and welcome.

These techniques contribute to the sample WMC in the following percentages:

$\begin{aligned} 95.8 \% & \text { visual binaries and optical pairs } \\ 50.6 \% & \text { interferometric binaries and optical pairs } \\ 1.7 \% & \text { spectroscopic binaries } \\ 1.4 \% & \text { cataclysmic variables or related objects } \\ 1.0 \% & \text { occultation binaries } \\ 0.3 \% & \text { astrometric binaries } \\ 0.2 \% & \text { eclipsing binaries } \\ 0.2 \% & \text { X-ray binaries } \\ 0.1 \% & \text { spectrum binaries } \\ 0.1 \% & \text { planets }\end{aligned}$

Since the techniques are complementary, the sum is $>100 \%$. It should be noted that this breakdown is biased significantly by selection effects. For example, while visual binaries may be discovered (and cataloged) after a single observation, data on spectroscopic pairs are often not published until the full orbit has been characterized.

\section{Coordinate Matching}

System matches are based on the arcsecond-precise coordinates of the primary stars in each individual pair. The most time-consuming aspect of the WMC construction (by far!) was the improvement of the arcminute-precise coordinates found in the WDS. Some $80 \%$ of the 1,645 different primaries in the sample region were matched to Hipparcos or Tycho-2 objects. An additional $19 \%$ matched to GSC2, USNO A2, 2MASS, etc. via individual inspection using ALADIN. There remain $1.3 \%$ (21 pairs) which still have only arcminute accuracy coordinates. Nearly all of these are older, unconfirmed, visual doubles (including some very wide common proper motion pairs and some with suspect coordinates).

Following coordinate matching we found a total of 1,465 systems in this slice of the sky. These may be broken down as follows:

$1,336(91 \%) \quad$ simple binaries

$80(5.5 \%)$ non-hierarchical triples

$16(1.1 \%)$ non-hierarchical systems, $>3$ components

$25(1.7 \%)$ hierarchical triples

$8(0.5 \%)$ hierarchical systems, $>3$ components 


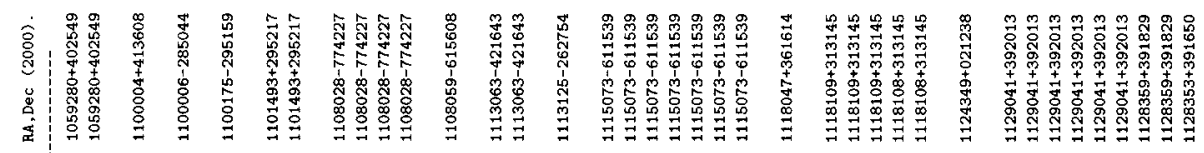

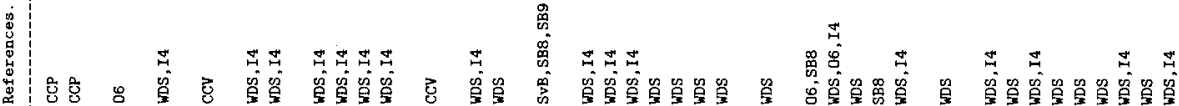

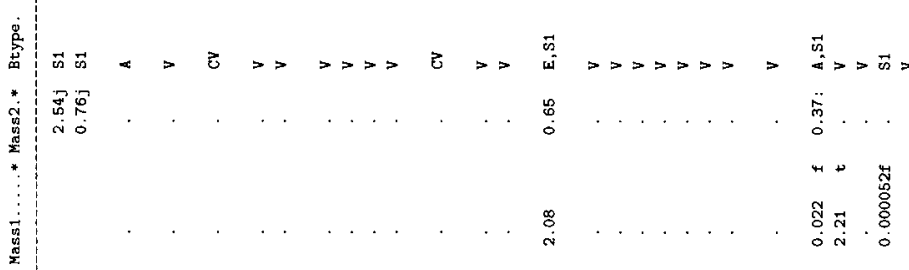

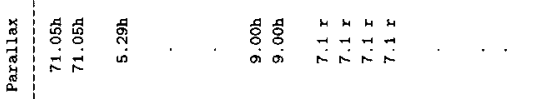

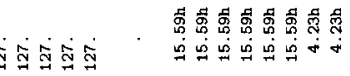

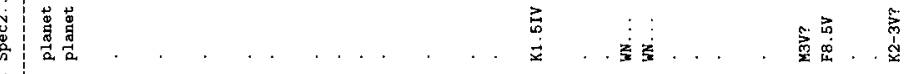

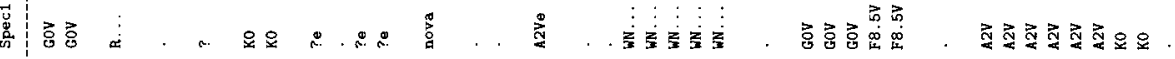

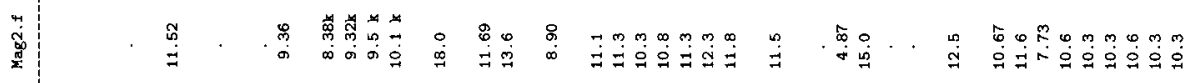

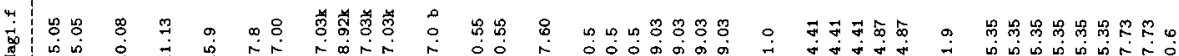

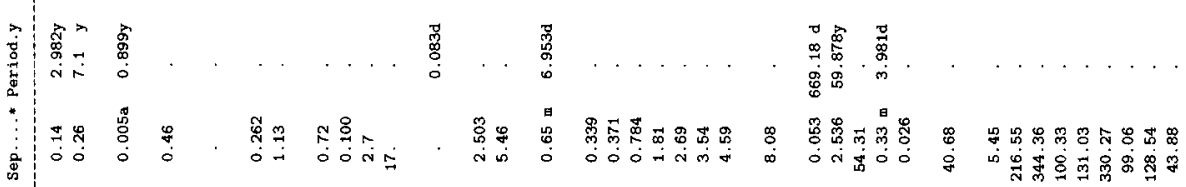

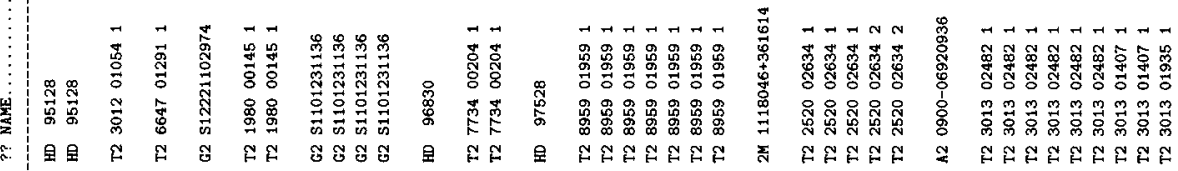

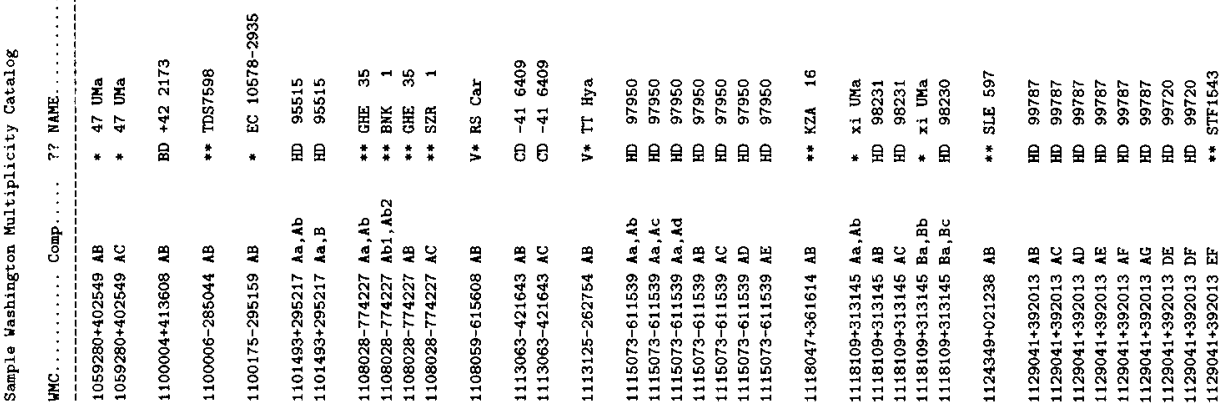

Figure 2. Sample page from the WMC Catalog. 


\section{The Catalog}

A sample page from the catalog is shown in Figure 2, and an explanation of the columns follows. (Note: In the explanation for columns 3 and 4 , the names in parentheses indicate the usual order of preference for that column.).

1 WMC designation (WDS or J2000 arcsecond coordinates of system primary)

2 component designation (AB; $\mathrm{Aa}, \mathrm{Ab}$; etc.)

3 catalog and name (Bayer/Flamsteed/variable star designation, HD, DM, discoverer designation, etc.)

4 catalog and name (HD, Tycho-2, GSC2, USNO A2, 2MASS, etc.)

5 angular separation (including separations predicted from spectroscopic orbit and parallax, vector separations, etc.)

6 orbital period

7,8 magnitudes (flags for variability, filter codes if not V)

9,10 spectral types

11 parallax (from Hipparcos, orbit, etc)

12,13 masses (or mass function, mass ratio. etc.)

14 binary type (visual, spectroscopic, X-ray, etc.) The 'visual' code will be modified if the pair is known to be optical, CPM, or physical.

15 references (with web links to on-line catalogs)

16 J2000 coordinates of principal star of pair $\left(0^{\prime \prime} .1\right.$ precision)

\section{Examples from the sample WMC}

A few systems from the portion of the sample WMC shown in Figure 2 are noted below. These illustrate some of the features of the WMC, as well as some of the difficulties to be encountered in creating the full catalog.

1100004+413608, 1100006-285044, etc.: These simple doubles, whether of visual, astrometric, spectroscopic, or other discovery origin, are by far the most common entries in the WMC.

1059280+402549 and 1113063-421643: These two triples are treated in the same manner, even though one consists of three stars and the other a star and its two planets. In both cases, the apparent separations of the $A B$ and $A C$ pairs are comparable, so all components are considered to be at the same hierarchy.

1101493+295217: This is an example of a simple hierarchical triple.

1108028-774227: This is the only system in the sample WMC showing all three levels of hierarchy.

1129041+392013: All components of this apparently small cluster are wide, so no hierarchical structure was assigned, despite the wide range in separation.

1118109+313145: The $\xi$ UMa system is another complicated multiple. The A component is a spectroscopic binary; the B component has also been split spectroscopically (with separation estimated as 0.33 milli-arcsecond). There is also a component of B resolved by speckle interferometry. Based on the separations, one would ordinarily assign the speckle pair the second level of hierarchy (e.g., $\mathrm{Ba}, \mathrm{Bb}$ ) and assume one of those components was split spectroscopically 
(e.g., Ba1,Ba2). However, we are familiar with the speckle pair and suspect the resolution may be spurious, as it has never been confirmed. We're thus reluctant to assign a different hierarchy to the very close pair.

1115073-611539: This is another apparent small cluster. Since the component we call $\mathrm{A}$ was later resolved into four stars, we assigned those stars to a second apparent hierarchical level.

\section{Discussion}

(Note: Discussions of both Mason's and Hartkopf's talks follow.)

SCHMITZ: How is it determined which is component A and which is B?

HARTKOPF: If masses are known (or inferred from, say, spectral type) the more massive star is the primary. If masses are not known, the brighter (preferentially in $\mathrm{V}$ ) is selected as the ' $\mathrm{A}$ ' component (although whether to use peak or median brightness in the case of a variable has not been determined). If all factors are equal, it is the one on the left! (just kidding...)

TURNER: Once an A always an A? Or is there a provision for changing which is an A based on new information?

HARTKOPF: Andrei and Dimitri's talks will present opposing viewpoints of this very topic.

SCARFE: Two questions and a comment: (1) How do we handle an SB whose "primary" is the star with the easily measurable spectrum? (2) Should you include all variable radial velocity objects, where the variation has no other known cause? (3) You need to enlist the help of a cataloger of eclipsing systems, to include the several thousand known.

MASON: (1) If it is an SSB and is the only component you 'see' then it is, by default, the primary. If it is a DSB and nothing is known regarding mass ratio then it would be the primary here as well. (2) Well, other indirect detection methods, like occultation, are included. I suppose they should be included as well; however, has anything listing just velocity variation been published since the Abt \& Biggs "Bibliography of Stellar Radial Velocities in 1972"? (3) We would gladly accept the help of any experts that might be willing to lend a hand.

SCARFE: Following Peter Eggleton's comment, it would be useful if authors would provide cross-references to the names of whole systems in the various catalogues in which they appear.

OSWALT: Some systems have several or even many different names. By what priority are the names listed in the WMC chosen?

HARTKOPF: There are two columns of designations. The order of priority in the first column is Bayer/Flamsteed or variable star designation $\rightarrow$ HD number $\rightarrow$ DM number $\rightarrow$ other catalog designation. For the second column we wanted to use a catalog which was as inclusive as possible so we mainly use the Tycho designation, which covers $80 \%$ of the catalog or more, then GSC2.2, A2, 2MASS, etc. 
MASON: Note that we only use discoverer designations in column one when nothing else is available.

OSWALT: Perhaps, following the example of the minor planet community, a provisional designation would be assigned to a binary. Then once physical association has been proved, a permanent designation assigned. Alternatively, a "provisional" flag in the catalog could be used for new systems.

HARTKOPF: In the column giving binary type we will indicate, when known, whether the system is an optical pair, a common-proper motion system, a physical system showing orbital motion, etc.

OSWALT: (1) (comment) The primary should probably be the one designated as such in the historical record - otherwise the literature will become confusing. (2) If for new binaries the mass determines which is designated primary, how will we deal with white dwarfs, mass transferring stars, or other stars where the star WAS the most massive, but is no longer?

HARTKOPF: I think we have to go with which is the most massive now, since our theories of evolution may be in error and change.

QUIRRENBACH: What is the approach regarding inclusion or exclusion of chance projections? If you go sufficiently deep, each star becomes "double". High-dynamic-range searches for low-mass companions are turning up lots of chance projections. One should have consistent criteria for what should be included.

MASON: Good point. Obviously we could go overboard and include everything, but we need to set some sort of limit before it becomes absurd. Setting the limit using Aitken's or Rossiter's criteria is a possibility but leaves out some certainly optical pairs that could be important for precise astrometry, blended images, etc.

SCHMITZ: Where will the central depository be for defining the A and B assignments?

HARTKOPF: The USNO Double Star group (i.e., us).

EVANS: Your catalog of "spectrum binaries" — would that include a Cepheid with a hot companion in the ultraviolet?

HARTKOPF: Yes. 